\title{
Percepção da população idosa sobre o HIV/AIDS: uma revisão integrativa
}

\author{
Perception of the elderly population on HIV/AIDS: na integrative review \\ Percepción de la población mayor sobre el VIH/SIDA: una revisión integrativa
}

Recebido: 21/09/2021 | Revisado: 30/09/2021 | Aceito: 03/10/2021 | Publicado: 05/10/2021

Lucas Mendes Feitosa Dias

ORCID: https://orcid.org/0000-0002-8706-9945

Universidade Federal do Piaú, Brasil

E-mail: lucarmendi@ufpi.edu.br

Mylenna Silva Crateús

ORCID: https://orcid.org/0000-0001-7458-4553 Universidade Estadual do Piauí, Brasil E-mail: mylennacrateus@gmail.com

Isabella Custodio da Silva Mota

ORCID: https://orcid.org/0000-0002-2445-4408 Universidade Federal do Piauí, Brasil E-mail: bell.custodio26@gmail.com

Bárbara Rebeca Alves Pereira

ORCID: https://orcid.org/0000-0001-5894-9161 Universidade Federal do Piaú, Brasil

E-mail: barbara rebeca66@hotmail.com

Waleska Ferreira de Albuquerque

ORCID: https://orcid.org/0000-0001-8775-8866 Universidade Federal do Piaú, Brasil E-mail: waleska@ufpi.edu.br

\begin{abstract}
Resumo
A AIDS é definida como uma infecção de natureza crônica resultante da ação do vírus HIV, que aumenta a vulnerabilidade do indivíduo a inúmeras doenças de caráter oportunista. A sociedade rotula a população idosa como assexuada ou não interessada em sexo, criando obstáculos que podem dificultar o enfrentamento. O trabalho tem como objetivo mostrar a percepção da população idosa sobre o HIV/AIDS, aspectos sociais envolvidos e o papel dos profissionais de saúde diante dessa temática. Trata-se de uma revisão integrativa da literatura nas bases de dados MEDLINE, BDENF, LILACS e SciELO em que adotou-se a estratégia PICO/PRISMA com o uso dos descritores: Aged, HIV, Knowledge. Os idosos estão cada vez mais convivendo com o HIV/AIDS, porém são vistos pelos demais como não praticantes de sexo, possuem uma vida sexual ativa e, há preferência pelo não uso do preservativo, principalmente em idosos do sexo masculino. O preservativo é visto apenas como contraceptivo em detrimento à prevenção. As mulheres estão mais susceptíveis a adquirir infecção pelo HIV por questões relacionadas à idade e cultura. Idosos habitantes de zonas rurais são ainda mais vulneráveis e possuem maiores dificuldades de assistência, comparado aos que vivem em zonas urbanas. Observa-se a necessidade da realização de ações mais direcionadas a saúde sexual dos idosos, solicitação de exames e fornecimento de preservativos femininos e masculinos. Faz-se necessário urgentemente a criação de políticas sobre a necessidade de planejar, orientar e implementar ações tendo em vista o empoderamento dos idosos frente a prevenção, intervenção e diagnóstico de HIV/ AIDS.
\end{abstract}

Palavras-chave: Idoso; HIV; Conhecimento.

\begin{abstract}
AIDS is defined as a chronic infection resulting from the action of the HIV virus, which increases the vulnerability of the individual to numerous diseases of an opportunistic nature. Society labels the elderly population as asexual or not interested in sex, creating obstacles that can make coping difficult. The work aims to show the perception of the elderly population about HIV/AIDS, social aspects involved and the role of health professionals in this regard. This is an integrative review of the literature in medline, BDENF, LILACS and SciELO databases in which the PICO/PRISMA strategy was adopted using the descriptors: Aged, HIV, Knowledge. The elderly are increasingly living with HIV/AIDS, but are seen by others as non-sex practitioners, have an active sexual life and, there is preference for not using condoms, especially in male elderly. Condoms are seen only as contraceptives to the detriment of prevention. Women are more likely to get HIV infection due to age and culture issues. Elderly inhabitants of rural areas are even more vulnerable and have greater difficulties in care, compared to those living in urban areas. It is observed the need to perform actions more directed to the sexual health of the elderly, request for tests and supply of female and male condoms. It is urgently necessary to create policies on the need to plan, guide and implement actions with a view to the empowerment of the elderly in the face of hiv/AIDS prevention, intervention and diagnosis.
\end{abstract}

Keywords: Aged; HIV; Knowledge. 


\begin{abstract}
Resumen
El SIDA se define como una infección crónica resultante de la acción del virus vih, que aumenta la vulnerabilidad del individuo a numerosas enfermedades de carácter oportunista. La sociedad etiqueta a la población anciana como asexual o no interesada en el sexo, creando obstáculos que pueden dificultar el afrontamiento. El trabajo tiene como objetivo mostrar la percepción de la población anciana sobre el VIH/SIDA, los aspectos sociales involucrados y el papel de los profesionales de la salud en este sentido. Se trata de una revisión integradora de la literatura en las bases de datos medline, BDENF, LILACS y SciELO en la que se adoptó la estrategia PICO/PRISMA utilizando los descriptores: Envejecimiento, VIH, Conocimiento. Los ancianos viven cada vez más con el VIH/SIDA, pero son vistos por otros como profesionales no sexuales, tienen una vida sexual activa $\mathrm{y}$, existe una preferencia por no usar condones, especialmente en los ancianos varones. Los condones son vistos sólo como anticonceptivos en detrimento de la prevención. Las mujeres son más propensas a contraer la infección por el VIH debido a problemas de edad y cultura. Los ancianos habitantes de las zonas rurales son aún más vulnerables y tienen mayores dificultades en la atención, en comparación con los que viven en las zonas urbanas. Se observa la necesidad de realizar acciones más dirigidas a la salud sexual de los ancianos, solicitud de pruebas y suministro de preservativos femeninos y masculinos. Es urgentemente necesario crear políticas sobre la necesidad de planificar, orientar y aplicar medidas con miras a la potenciación de las personas de edad frente a la prevención, la intervención y el diagnóstico del VIH/SIDA.
\end{abstract}

Palabras clave: Envejecido; VIH; Conocimiento.

\title{
1. Introdução
}

A AIDS (Síndrome da Imunodeficiência Adquirida) é definida como uma infecção de natureza crônica resultante da ação do vírus HIV (Vírus da Imunodeficiência Humana), que diminui a resposta imune mediada pelos linfócitos TCD4+, aumentando a vulnerabilidade do indivíduo a inúmeras doenças de caráter oportunista. Essa síndrome afeta uma parcela considerável da população mundial, independente da raça, cultura e aspectos políticos e sociais. Incluindo os idosos, que se encontram vulneráveis. (De Souza et al, 2018; Tayni et al, 2018).

A síndrome da imunodeficiência adquirida (AIDS) foi evidenciada primeiramente no ano de 1981 a partir da ocorrência de doenças imunológicas ligadas à disfunção sexual observados nos Estados Unidos (EUA). Ao analisar a epidemiologia da doença em tempos atuais verifica-se que os idosos surgem são população de risco em potencial, pelo fato dos estudos revelarem que a aumento nos casos de HIV em pessoas com mais de 50 anos estão infectadas pelo HIV, e a população está envelhecendo cada vez mais. Existem inúmeros fatores que estão relacionados à fragilidade da população idosa ao HIV/AIDS, entre eles podemos destacar: ausência de assistência profissional, carência de campanhas de conscientização ao comportamento sexual, uma vez que a sexualidade dessa população, muitas vezes é cheia de tabus e preconceitos sociais, podendo causar suspeitas e inseguranças (Silva et al, 2015; Brito et al, 2016; Cassete et al, 2016).

Tanto as informações sobre a AIDS e outras ISTs (infecções sexualmente transmissíveis) como as políticas de educação sexual são voltadas em sua grande maioria ao público jovem-adulto, o que pode ocasionar um impacto negativo na busca de idosos, como aconselhamento e testagem. A sociedade tem o hábito de rotular a população idosa como assexuada ou não interessada em sexo, o que leva a criação de obstáculos relacionados ao assunto que podem ser influenciados pelo estado civil dos idosos. Além do mais, como a gravidez está fora de questão nesse caso, acabam não fazendo a utilização do preservativo (Branton et al, 2016; Moreli-Mengual et al, 2018; Bittencourt et al, 2015; Taylor et al, 2017).

A OMS e o Ministério da Saúde reconhecem tanto a necessidade como a importância de falar sobre o comportamento sexual, não só pelo que já foi mencionado, mas também por ser um tópico essencial na vida dessa população. Além disso, o público idoso não atendido seja por razões sociais ou econômicas, em certos períodos da vida como o início da vida sexual, podem não saber da existência de auxílio profissional e podem resistir à assistência profissional, dificultando a resolução do problema. (Anjos et al, 2016; Simpson et al, 2017). Diante disso, o objetivo do trabalho trata-se de mostrar por meio de revisão bibliográfica a percepção da população idosa sobre o HIV/AIDS, aspectos sociais envolvidos e o papel dos profissionais de saúde diante dessa temática. 


\section{Metodologia}

Trata-se de uma revisão integrativa. Este método de pesquisa consiste em um levantamento bibliográfico que proporciona a síntese de conhecimento e a incorporação da aplicabilidade de resultados de estudos significativos na prática (Souza; Silva; Carvalho, 2010). Fora elaborado a partir das seis etapas, sendo estas (Mendes; Silveira; Galvão, 2008):

1.Identificação do tema e seleção da hipótese ou questão de pesquisa para a elaboração da revisão integrativa;

2.Estabelecimento de critérios para inclusão e exclusão de estudos/ amostragem ou busca na literatura;

3.Definição das informações a serem extraídas dos estudos selecionados/ categorização dos estudos;

4.Avaliação dos estudos incluídos na revisão integrativa;

5.Interpretação dos resultados;

6.Apresentação da revisão/síntese do conhecimento

Para elaboração da pergunta norteadora fora utilizada PICO. Esta estratégia é utilizada para auxiliar na construção da pergunta da pesquisa a partir do uso de elementos fundamentais para guiar a busca bibliográfica baseada em evidências, sendo estes: Paciente, Intervenção, Comparação e "Outcomes" (desfecho) (Santos; Pimenta; Nobre, 2007). O fluxo deste método está descrito no Quadro 1.

Quadro 1 - Acrônimos para a elaboração da pergunta norteadora conforme a estratégia PICO.

\begin{tabular}{|cc|}
\hline P & "IDOSOS” \\
I & "PERCEPÇÃO SOBRE O HIV/AIDS" \\
C & NÃO SE APLICA \\
O & CONHECIMENTO \\
\hline
\end{tabular}

Fonte: Elaborada pelos autores (2020).

A busca ocorreu entre junho e julho de 2020. Foram utilizados como bancos de dados MEDLINE (Medical Literature Analysis and Retrieval System Online), BDENF (Base de dados em Enfermagem), LILACS (Latin American and Caribbean Health Sciences Literature) e SciELO (Scientific Eletronic Library Online). Para a realização da busca, foram escolhidos os seguintes descritores, ambos cadastrados no DeCS (Descritores em Ciências da Saúde) e MeSH (Medical Subject Headings): Aged, HIV, Knowledge. Os critérios de inclusão e exclusão estão descritos logo abaixo.

- Critérios de inclusão: Estar publicado entre 2013 a 2020; Estar publicado em inglês, espanhol, francês ou português; Estar disponível para leitura em texto completo.

- $\quad$ Critérios de exclusão: Estudos que trabalhassem com uma população alvo com idade inferior a 60 anos; Estudos que descrevessem manifestações clínicas não relacionadas ao HIV/AIDS.

O fluxograma de busca apresenta-se descrito na Figura 1, seguindo a recomendação PRISMA. 
Figura 1 - Fluxograma de pesquisa conforme a recomendação PRISMA.

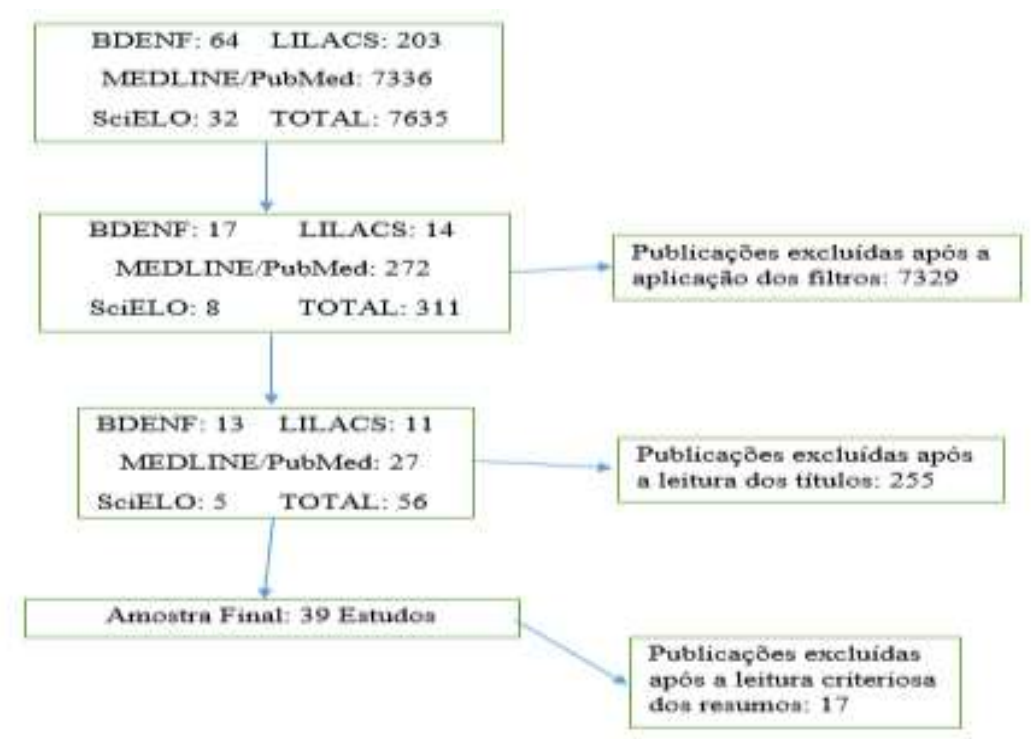

Fonte: Elaborada pelos autores (2020).

\section{Resultados e Discussão}

As discussões relacionadas à infecção pelo HIV não estão reclusas apenas em grupos de jovens e adultos. Longe disso, a literatura destaca que os idosos estão cada vez mais convivendo com o HIV/AIDS. Essa população cresce independentemente da situação socioeconômica do local onde resida. Isto gera discussões sobre fragilidades e possíveis lacunas que práticas de educação em saúde devem ser constantemente atualizadas de acordo com as peculiaridades dos indivíduos (Bastos et al, 2018; Poynten et al, 2013).

Esta problemática pode ser justificada por estereótipos criados pela sociedade. O idoso por estar em uma idade mais avançada e possuir mais dificuldade em realizar tarefas é visto pelos demais como um indivíduo que não realiza prática sexual e nem costuma pensar neste ato. Entretanto, percebeu-se que a maioria dos idosos possuem uma vida sexual ativa e, mesmo conhecendo os riscos que uma relação desprotegida possa causar, há preferência pelo não uso deste método o que torna passível a transmissibilidade do HIV/AIDS. (Malaquias et al, 2017; Uchôa et al, 2016).

Apesar das diminuições na capacidade física e mudanças fisiológicas ocasionadas pelo envelhecimento, a sexualidade faz parte da vida do idoso, o preconceito social em relação à sexualidade na terceira idade traz uma percepção do idoso como um ser assexuado, porém a literatura alega que a maioria dos idosos mantém o interesse sexual e praticam sexo. A ausência de parceiro fixo é uma das causas de falta de atividade sexual para vários idosos, porém existem idosos que têm vários parceiros sexuais e às vezes parceiros mais jovens (Santos et al, 2014; Bittencourt et al, 2015).

Os estudos demonstram que apesar da maioria dos idosos ter conhecimento sobre a transmissão do HIV e o uso do preservativo como método de prevenção às infecções sexuais, muitos deles não praticam sexo seguro e ainda acredita em mitos sobre o vírus (Brito et al, 2016; Altschuler et al, 2017). Existe uma certa resistência ao uso do preservativo, principalmente no que diz respeito aos idosos do sexo masculino por constrangimento na hora de adquirir o preservativo, ou por desconhecimento de como usá-lo. Em relação às idosas, observou-se que há uma dificuldade em convencer o parceiro a usar o preservativo. Além disso, por não haver reforço na mídia sobre a infecção do HIV/AIDS em idosos, estes não acreditam ser um grupo vulnerável a este vírus e, por isso não utilizam camisinhas (Caetano et al, 2018).

Houve mudanças no manejo de certas doenças quando feita comparação com o século passado. A exemplo disso, temse o HIV/AIDS que no século XX seu tratamento era associado a uma situação desgastante que muitas das vezes possuíam um 
final trágico. Atualmente, com a melhoria dos tratamentos e intervenções em saúde, observou-se que houve distanciamento da associação do HIV/AIDS com a morte, tornando os métodos de prevenção banalizados, muitas vezes não utilizados por acreditar no tratamento existente (Nardelli et al, 2016; Brandao et al, 2019).

A falta de informação sobre o HIV/AIDS na população idosa causa uma dificuldade no manejo desse indivíduo. Cabe o profissional de saúde utilizar seus conhecimentos sobre a temática para que oriente o idoso sobre medidas de prevenção do HIV/AIDS e as consequências passíveis de ocorrer caso ele se infecte com esse vírus (Alwafi et al, 2018; Morillo Verdugo et al, 2018). Vale ressaltar que a orientação deve ser de acordo com o nível educacional do indivíduo, pois durante esta faixa etária existe dificuldade no repasse de informações por permanência do conhecimento de situações vivenciadas por ele (Costa et al, 2018; Nardelli et al, 2019).

Concomitante com isso, observa-se que idosos que ao decorrer de sua vida tenham realizado testes para HIV ou que possuam algum familiar que conviva com esta infecção tendem a conhecer os riscos que o HIV/AIDS possa trazer a ele, assim como medidas de prevenção e tratamento (Singo et al, 2015). Além disso, idosos em situação de primeiro sexo casual conhecem os riscos e em decorrência disso, utilizam formas de prevenção com maior frequência como, por exemplo, preservativo. Um fator relevante corresponde a estabilidade da relação. Observou-se que idosos que possuem relacionamentos estáveis e tenham tido experiências com o HIV/AIDS como realização de testes de HIV ou algum familiar que tenha esta infecção (Souza et al, 2016; Nascimento et al, 2017; Saggiorato et al, 2015)

Há recomendações na literatura que mostram que a utilização de recursos audiovisuais funciona como uma forma de aproximar o idoso e a temática do HIV/AIDS. Uma das estratégias utilizadas pelos autores é a exibição de filmes que mostravam generalidades sobre o tema, tais como: prevenção, mudanças no estilo de vida em decorrência do HIV/AIDS, além do reforço sobre a importância da adesão ao tratamento. Percebeu-se que os indivíduos que estiveram presentes durante esta estratégia sentiram-se mais íntimos com o tema e, consequentemente, mais preparados para conviver ou prevenir esta infecção (Ebor et al, 2015; Anjos et al, 2016).

No que diz respeito aos agentes causadores de HIV na melhor idade, os estudos mostraram que a mudança nos padrões de comportamento da população idosa, a falta de informação e de políticas públicas destinadas à prevenção do HIV/AIDS em idosos juntamente com o machismo e outros aspectos culturais cumprem papel importante no aumento da vulnerabilidade e de casos na população idosa, observou-se também que as mulheres além de serem mais vulneráveis ainda à exposição, são sempre maioria nos estudos epidemiológicos relacionados ao assunto. Em relação a utilização do preservativo, a literatura mostra que apesar de muitos idosos conhecerem os métodos preservativos, ainda há bastante resistência quanto ao seu uso e a vida heterossexual é a principal via de transmissão do HIV/AIDS em idosos (Santos et al, 2018; Silva et al, 2017).

O aumento de espaços e locais de convivência, aumento da longevidade e fácil acesso a medicamentos para disfunção erétil, tornaram possíveis a criação de relações afetivas ao idoso, possibilitando a formação de mais laços afetivos, deixando de ser "assexuado". Considera-se que não é a expressão da sexualidade em si que gera o aumento da vulnerabilidade da população idosa ao HIV/AIDS e sim a prática sexual sem proteção e de forma irresponsável, diante disso é necessário que o sistema de saúde forneça mecanismos para que esse grupo possa adotar práticas sexuais de maneira segura. Devido a maior facilidade de expressão da sexualidade nos tempos atuais, os idosos tornaram-se vulneráveis ao HIV/Aids, sendo um desafio para os profissionais de saúde na criação de medidas de informação e proteção desse grupo (Burigo et al, 2015; Uchôa et al, 2016).

Um estudo realizado por Bezerra et al. (2015) com 84 idosos na cidade de João Pessoa - PB em 2012 mostrou que mostra que $85,7 \%$ dos idosos reconhecem o uso do preservativo como um método de proteção contra doenças e 75,0\% como um recurso necessário nas relações sexuais, mas ao mesmo tempo 60,7\% acreditam que uso provoca desconfiança no casal e ser desnecessário em casos de parceiro fixo/união estável. A literatura revela que o uso dos preservativos entre idosos, ainda encontra dificuldades, devido a crenças que levam a acreditar que tal uso possa interferir no prazer e ereção, constituindo-se também que 
a prática do uso pode ser atribuída como sinônimo de infidelidade no relacionamento (Lekalakala-Mokgele et al, 2014). Os idosos enxergam o preservativo apenas como meio contraceptivo em detrimento à prevenção, contribuindo para a prática de um sexo sem segurança. Vale destacar também um fator importante para o não uso do preservativo, é a ideia de que homens e mulheres em relacionamentos estáveis não há risco de infecção. Esta ideia de imunidade, relacionada à confiança, amor romântico, é mais presente em mulheres que possuem menor percepção da sua condição de vulnerabilidade. Por isso, ao sugerir a utilização do preservativo para não contracepção, pode provocar a desconfiança do parceiro (Ribeiro et al, 2019; Ferreira et al, 2019).

A desigualdade entre homens e mulheres é resultado de um processo histórico que mostra uma situação de inferioridade da mulher em relação ao homem, uma vez que as mulheres não tinham poder de decisão e sofriam violência de inúmeras formas. Tudo isso corroborou para uma menor liberdade sexual e menos poder de decisão acerca do sexo com proteção. Desta forma, as relações desiguais entre os sexos se traduzem em maior vulnerabilidade para as mulheres ao contágio pelo HIV (Aguiar et al, 2020; Aguiar et al, 2020).

Segundo Lekalakala-Mokgele et al. (2016) as mulheres estão mais susceptíveis a adquirir infecção pelo HIV devido a questões relacionadas à idade e cultura uma vez que costumam culpar as infidelidades do parceiro e logo após perdoá-las. E para completar, muitos homens casados continuavam a manter relações sexuais com seus cônjuges e parceiros mais jovens, aumentando o risco de contrair e transmitir o HIV. Foi afirmado também que as mulheres sentiam que precisavam agradar seus parceiros a qualquer custo, satisfazendo suas necessidades sexuais, independentemente de seus próprios desejos sexuais. Isso está diretamente relacionado a medos e relacionamentos submissos com parceiros do sexo masculino, tornando menos possível e negociação e adoção das medidas de proteção para prevenir a infecção pelo HIV (Li Ning et al, 2017).

Para o rastreio e diagnóstico vírus, faz-se necessário a utilização de dados laboratoriais acerca do vírus HIV (como contagens de células T CD4 + e cargas virais) e, dependendo da gestão e da metodologia empregada esses dados podem ser confusos e imprecisos, sendo um ponto negativo na hora do diagnóstico pois são utilizados como indicadores de saúde do paciente (Gakumo et al, 2017). Para que haja uma comunicação efetiva e sem danos para o paciente é crucial que haja sensibilidade e compreensão por parte do provedor da notícia, pois esse paciente pode apresentar limitações que podem afetá-lo, muitas vezes relacionadas com analfabetismo, por exemplo, ou ainda analfabetismo em saúde (o grau em que os indivíduos têm capacidade de acesso, processar, interpretar, comunicar e agir de forma numérica, quantitativa, gráfica, bioestatística e probabilística informações de saúde necessárias para tornar decisões de saúde) (Rosenfeld et al, 2016; O’Brien et al, 2014).

Além do mais, idosos habitantes de zonas rurais são ainda mais vulneráveis e possuem maiores dificuldades de assistência, comparado aos que vivem em zonas urbanas. Isso é resultado dos desajustes socioeconômicos, incluindo falta de transporte, acesso limitado a instalações, falta de serviços de saúde e recursos para o tratamento do HIV nessas áreas (Singo et al, 2015; Brandao et al, 2019). Outro fator importante, é discriminação e preconceito em relação a portadores de HIV, o que pode levar a um quadro depressivo, isolamento social, e no caso de idosos, pode levar também a diminuição da mobilidade, pois o idoso se abstém de sair e cumprir atividade como ir ao supermercado ou outros lugares de convívio por vergonha, ou ainda, por falta de informação quanto a forma de transmissão, por exemplo (Slater et al, 2013; Wallace et al, 2017).

É papel dos profissionais de saúde proporcionarem o acesso a informação sobre o HIV/AIDS, maneiras de transmissão e prevenção para a população, com o intuito de minimizar os paradigmas e, consequentemente, a discriminação. Essa informação deverá ser passada de maneira simples e explicativa de acordo com o público, no caso dos pacientes, o profissional deve construir um canal de comunicação mútico e efetivo (Nascimento et al, 2017; Schatz et al, 2019).

\section{Considerações Finais}

Diante dos resultados e partindo da avaliação das intervenções e perspectivas acerca da temática do estudo, constata-se 
a necessidade da realização de ações mais direcionadas a saúde sexual dos idosos e o seguimento do trabalho com a solicitação de exames e fornecimento de preservativos femininos e masculinos. Neste sentido, faz-se necessário urgentemente a criação de políticas mais eficazes e, sobretudo uma conscientização dos profissionais de saúde sobre a necessidade de planejar, orientar e implementar ações tendo em vista o empoderamento dos idosos frente a prevenção, intervenção e diagnóstico de HIV/ AIDS.

As ações neste sentido devem ser constantes e inovadoras, buscando integrar a comunidade, os idosos e sua realidade nos planejamentos das políticas de saúde para as unidades de saúde da Família. É enganoso pensar que o sexo só pode ser vivenciado pelos jovens, e que o idoso não precisa disso, é um ser assexuado. Porém, a sexualidade deve ser vivenciada e experimentada em todas as fases da vida, e depende dos estímulos e desejos que cada indivíduo vivencia. Diante disso, pode-se inferir que a troca de experiências através da educação em saúde, seria fundamental para tentar estimular os idosos a refletir sobre sua sexualidade e vivenciá-la de forma mais natural.

Uma vez que os estereótipos sociais e os preconceitos são elementos da cultura e, portanto, contribuem no processo de formação dos indivíduos e no conjunto de relações sociais, parece fundamental que tais discussões sejam incluídas ao longo do processo de formação em saúde, tanto na graduação quanto nas ações de formação permanente dos profissionais de saúde, com vistas a compreender a importância desses elementos nos processos de saúde e adoecimento e na promoção de saúde. A visão sobre a velhice e o envelhecimento; as temáticas da sexualidade e das práticas sexuais, especialmente em idosos, seguem como desafios para a atuação em saúde e promoção da saúde da pessoa idosa. Além disso, percebe-se que o diagnóstico de HIV em idosos, em seu potencial estigmatizante, está vinculado às experiências de sofrimento do sujeito em relação à visão que tem de si e das formas como será percebido e julgado pelos outros; das possibilidades de tratamento e promoção de saúde. Sendo assim, cabe destacar que, pelas características específicas de pesquisas qualitativas analisadas, as análises empreendidas não buscam estabelecer generalizações em relação à complexidade da situação analisada, tampouco a pretensão de esgotar as discussões sobre os temas aqui abordados.

Os resultados desta pesquisa podem contribuir na reformulação da organização do processo de trabalho em saúde, das práticas profissionais, sustentar ações de formação permanente, subsidiar políticas públicas sobre a promoção de saúde da pessoa idosa e lançar novos olhares aos problemas enfrentados. Enfatiza-se a necessidade de desdobramentos de estudos que consigam aprofundar na interferência dos estereótipos, estigmas e preconceitos nas práticas em saúde.

Através de medidas educativas desenvolvidas por profissionais capacitados é possível proporcionar mudanças comportamentais nos idosos principalmente no que diz respeito às formas de transmissão e medidas de prevenção ao HIV/AIDS. É crucial também o desenvolvimento de mais estudos epidemiológicos voltados para o nível de conhecimento dos idosos sobre o HIV/AIDS, número de casos diagnosticados anualmente nessa população e os fatores desencadeantes.

\section{Referências}

Aguiar, R. B., Leal, M. C. C., \& Marques, A. P. D. O. (2020). Conhecimento e atitudes sobre sexualidade em pessoas idosas com HIV. Ciência \& Saúde Coletiva, 25, 2051-2062.

Aguiar, R. B., Leal, M. C. C., Marques, A. P. D. O., Torres, K. M. S., \& Tavares, M. T. D. B. (2020). Idosos vivendo com HIV-comportamento e conhecimento sobre sexualidade: revisão integrativa. Ciência \& Saúde Coletiva, 25, 575-584.

Altschuler, J. (2017). Midlife and older women's experiences and advice about sex with men, risk behaviors, and HIV prevention education. Journal of women \& aging, 29(1), 63-74.

Alwafi, H. A., Meer, A. M., Shabkah, A., Mehdawi, F. S., El-Haddad, H., Bahabri, N., \& Almoallim, H. (2018). Knowledge and attitudes toward HIV/AIDS among the general population of Jeddah, Saudi Arabia. Journal of infection and public health, 11(1), 80-84.

Anjos, K. F. D., Oliveira, A. C., Suto, C. S. S., Guimarães, F. E. D. O., Sobrinho, C. L. N., \& Rosa, D. D. O. S. (2016). Bioethical issues involved in care of elderly with HIV/AIDS. Revista de Pesquisa: Cuidado é Fundamental Online, 8(3), 4882-4890.

Bastos, L. M., Tolentino, J. M. S., Frota, M. A. D. O., Tomaz, W. C., Fialho, M. L. D. S., Batista, A. C. B., \& Barbosa, F. C. B. (2018). Evaluation of the level of knowledge about Aids and syphilis among the elderly from a city in the interior of the state of Ceará, Brazil. Ciencia \& saude coletiva, 23(8), $2495-2502$. 
Bezerra, V. P., Serra, M. A. P., Cabral, I. P. P., Moreira, M. A. S. P., Almeida, S. A. D., \& Patrício, A. C. F. D. A. (2015). Práticas preventivas de idosos e a vulnerabilidade ao HIV. Revista Gaúcha de Enfermagem, 36, 70-76.

Bittencourt, G. K. G. D., Moreira, M. A. S. P., Meira, L. C. D. S., Nóbrega, M. M. L. D., Nogueira, J. A., \& Silva, A. O. (2015). Concepções de idosos sobre vulnerabilidade ao HIV/Aids para construção de diagnósticos de enfermagem. Revista Brasileira de Enfermagem, 68, 579-585.

Brandon, M. (2016). Psychosocial aspects of sexuality with aging. Topics in Geriatric Rehabilitation, 32(3), 151-155.

Brandão, B. M. G. D. M., Angelim, R. C. D. M., Marques, S. C., Oliveira, D. C. D., Oliveira, R. C. D., \& Abrão, F. M. D. S. (2019). Representaciones sociales de ancianos soropositivos acerca del VIH/SIDA. Revista Brasileira de Enfermagem, 72, 1349-1355.

Burigo, G. D. F., Fachini, I. H., Garetti, B., Streicher, C. C. I., \& Rosa, R. S. (2015). Sexualidade e comportamento de idosos vulneráveis a doenças sexualmente transmissíveis. CuidArte, Enferm, 148-153.

Cassétte, J. B., Silva, L. C. D., Felício, E. E. A. A., Soares, L. A., Morais, R. A. D., Prado, T. S., \& Guimarães, D. A. (2016). HIV/AIDS among the elderly: stigmas in healthcare work and training. Revista Brasileira de Geriatria e Gerontologia, 19, 733-744.

Caetano, K. S., Oliveira, G. K. A., Santos, G. F., de Sá Barros, P., de Souza, M. R., \& Borges, C. J. (2018). HIV/AIDS: conhecimento, atitude e prática da pessoa idosa. Itinerarius Reflectionis, 14(4), 01-21.

Costa, M. S., Moreira, M. A. S. P., Silva, A. O., Leite, E. D. S., Silva, L. M., \& Sampaio, J. B. (2018). Knowledge, beliefs, and attitudes of older women in HIV/AIDS prevention. Revista brasileira de enfermagem, 71, 40-46.

Caliari, J. D. S., Reinato, L. A. F., Pio, D. P. M., Lopes, L. P., Reis, R. K., \& Gir, E. (2018). Qualidade de vida de idosos vivendo com HIV/aids em acompanhamento ambulatorial. Revista Brasileira de Enfermagem, 71, 513-522.

da Silva Santos, Á., Arduini, J. B., Silva, L. C., \& Fonseca, A. S. (2014). Compreensão de idosos e familiares sobre sexualidade e HIV/Aids: estudo descritivo. Online Brazilian Journal of Nursing, 13(2), 175-185.

de Brito, N. M. I., da Costa Andrade, S. S., da Silva, F. M. C., Fernandes, M. R. C. C., Brito, K. K. G., \& dos Santos Oliveira, S. H. (2016). Idosos, infecções sexualmente transmissíveis e AIDS: conhecimentos e percepção de risco. ABCS Health Sciences, 41(3).

de Oliveira Ferreira, C., Davoglio, R. S., Vianna, A. D. S. A., da Silva, A. A., de Rezende, R. E. A., \& Davoglio, T. R. (2019). Vulnerabilidade a infecções sexualmente transmissíveis em idosos usuários de um centro de testagem e aconselhamento. Arquivos de Ciências da Saúde da UNIPAR, 23(3).

Duarte de Souza, M. D. D., Macêdo Mota, L. I., Noleto dos Santos, W., Rosendo da Silva, R. A., \& Lima Monte, N. (2016). Knowledge Of The Elderly From The Family Health Strategy In Relation To Hiv/Aids. Journal of Nursing UFPE/Revista de Enfermagem UFPE, 10(11).

Ebor, M., Murray, A., Gaul, Z., \& Sutton, M. (2015). HIV Awareness and knowledge among viewers of a documentary film about HIV among racial-or ethnicminority older adults. Health \& social work, 40(3), 217-224.

Gakumo, C. A., et al. A Qualitative Study on Health Numeracy and Patient-Provider Communication of Laboratory Numbers in Older African Americans with HIV. Journal of the Association of Nurses in AIDS Care, 27(6), 826-834, 2016

Lekalakala-Mokgele, E. (2016). Exploring gender perceptions of risk of HIV infection and related behaviour among elderly men and women of Ga-Rankuwa, Gauteng Province, South Africa. Sahara-J: Journal Of Social Aspects Of Hiv/Aids, 13(1), 88-95.

Lekalakala-Mokgele, E. (2014). Understanding of the risk of HIV infection among the elderly in Ga-Rankuwa, South Africa. SAHARA-J: Journal of Social Aspects of HIV/AIDS, 11(1), 67-75.

Li, N., Li, H., Ma, Y. M., Fan, P. Y., Yang, W. J., \& Zhu, Q. (2017). A qualitative study on high risk behaviors and related factors of reported HIV/AIDS cases aged 60 years and above in some areas of Henan province. Zhonghua liu xing bing xue za zhi= Zhonghua liuxingbingxue zazhi, 38(9), 1161-1164.

Malaquias, B. S. S., Nardelli, G. G., Azevedo, N. F., Ledic, C. S., Gaudenci, E. M., \& Santos, Á. D. S. (2017). Sexuality and knowledge about hiv/aids in elders who participate in a social center for the elderly. Biosci. j.(Online), 465-475.

Mendes, K. D. S., Silveira, R. C. D. C. P., \& Galvão, C. M. (2008). Revisão integrativa: método de pesquisa para a incorporação de evidências na saúde e na enfermagem. Texto \& contexto-enfermagem, 17, 758-764.

Morillo Verdugo, Ramón A. et al. The challenge of aging and pharmacoterapeutic complexity in the HIV+ patient. Farmacia Hospitalaria, 42 (3), $120-127$. 2018 .

Nardelli, G. G., Malaquias, B. S. S., Gaudenci, E. M., Ledic, C. S., Azevedo, N. F., Martins, V. E., \& Santos, Á. D. S. (2017). Conhecimento sobre síndrome da imunodeficiência humana de idosos de uma unidade de atenção ao idoso. Revista Gaúcha de Enfermagem, 37.

Nardelli, G. G., et al. Knowledge about HIV/AIDS in older adults using the services of Family Health Strategy. Revista da Sociedade Brasileira de Medicina Tropical, v. 52, 2019.

Nascimento, E. K. S., Albuquerque, L. P. D. A., Marinelli, N. P., Campelo, M. N. A. A., \& Santos, F. J. L. D. (2017). História de vida de idosos com HIV/Aids. Rev. enferm. UFPE on line, 1716-1724.

O'Brien, K. K., Solomon, P., Trentham, B., MacLachlan, D., MacDermid, J., Tynan, A. M., \& Zack, E. (2014). Evidence-informed recommendations for rehabilitation with older adults living with HIV: a knowledge synthesis. BMJ open, 4(5), e004692.

Poynten, I. M., Grulich, A. E., \& Templeton, D. J. (2013). Sexually transmitted infections in older populations. Current opinion in infectious diseases, 26(1), 80-85. 
Research, Society and Development, v. 10, n. 13, e78101320892, 2021

(CC BY 4.0) | ISSN 2525-3409 | DOI: http://dx.doi.org/10.33448/rsd-v10i13.20892

Rosenfeld, D., Ridge, D., Catalan, J., \& Delpech, V. (2016). Age and life course location as interpretive resources for decisions regarding disclosure of HIV to parents and children: findings from the HIV and later life study. Journal of Aging Studies, 38, 81-91.

Ribeiro, Í. A. P., de Oliveira Dantas, R., da Silva Oliveira, A. D., Lago, E. C., \& Viana, F. C. R. (2019). Conhecimento e uso do preservativo por idosos na prevenção do HIV/Aids: Nota Prévia. Revista Enfermagem Atual In Derme, 88(26).

Saggiorato, A. K. S., \& Trevisol, F. S. (2015). Percepções sobre AIDS e comportamento sexual em idosos da cidade de Tubarão, Santa Catarina. DST j. bras. doenças sex. transm, 29-34.

Santos, C. M. D. C., Pimenta, C. A. D. M., \& Nobre, M. R. C. (2007). A estratégia PICO para a construção da pergunta de pesquisa e busca de evidências. Revista Latino-Americana de Enfermagem, 15, 508-511.

Santos, J. D. S. (2018). Conhecendo a vulnerabilidade ao HIV/AIDS de dois grupos de idosos.

Schatz, E., Seeley, J., Negin, J., Weiss, H. A., Tumwekwase, G., Kabunga, E., \& Mugisha, J. (2019). "For us here, we remind ourselves": strategies and barriers to ART access and adherence among older Ugandans. BMC Public Health, 19(1), 1-13.

Silva, A. O., de Loreto, M. D. D. S., \& Mafra, S. C. T. (2017). HIV na terceira idade: repercussões nos domínios da vida e funcionamento familiar. Revista Em Pauta: teoria social e realidade contemporânea, 15(39).

Simpson, P., Brown Wilson, C., Brown, L. J., Dickinson, T., \& Horne, M. (2017). The challenges and opportunities in researching intimacy and sexuality in care homes accommodating older people: a feasibility study. Journal of Advanced Nursing, 73(1), 127-137.

Singo, V. J., Lebese, R. T., Nemathaga, L. H., \& Maluleke, T. X. (2015). The views of the elderly on the impact that HIV and AIDS has on their lives in the Thulamela Municipality, Vhembe District, Limpopo province. Curationis, 38(1), 1-8.

Slater, L. Z., Moneyham, L., Vance, D. E., Raper, J. L., Mugavero, M. J., \& Childs, G. (2013). Support, stigma, health, coping, and quality of life in older gay men with HIV. Journal of the Association of Nurses in AIDS Care, 24(1), 38-49.

Souza, M. T. D., Silva, M. D. D., \& Carvalho, R. D. (2010). Integrative review: what is it? How to do it?. Einstein (São Paulo), 8, $102-106$.

Uchôa, Y. D. S., Costa, D. C. A. D., Silva, I. A. P. D., Silva, S. D. T. S. E. D., Freitas, W. M. T. D. M., \& Soares, S. C. D. S. (2016). A sexualidade sob o olhar da pessoa idosa. Revista brasileira de geriatria e gerontologia, 19, 939-949.

Wallace, L. M., Ferrara, M., Brothers, T. D., Garlassi, S., Kirkland, S. A., Theou, O., \& Guaraldi, G. (2017). Lower frailty is associated with successful cognitive aging among older adults with HIV. AIDS research and human retroviruses, 33(2), 157-163.

Zhang, T., Miao, Y., Li, L., \& Bian, Y. (2019). Awareness of HIV/AIDS and its routes of transmission as well as access to health knowledge among rural residents in Western China: a cross-sectional study. BMC public health, 19(1), 1-11. 